\title{
FINITE SIZE SCALING OF THE TYPICAL DENSITY OF STATES OF DISORDERED SYSTEMS WITHIN THE KERNEL POLYNOMIAL METHOD
}

\author{
DANIEL JUNG
}

School of Engineering and Science, Jacobs University Bremen gGmbH, Campus Ring 1, 28759 Bremen, Germany

d.jung@jacobs-university.de

GERD CZYCHOLL

Institute for Theoretical Physics, University of Bremen, Otto-Hahn-Allee 1, 28359 Bremen, Germany

czycholl@itp.uni-bremen.de

STEFAN KETTEMANN

School of Engineering and Science, Jacobs University Bremen gGmbH, Campus Ring 1, 28759 Bremen, Germany

Division of Advanced Materials Science, Pohang University of Science and Technology, San 31, Hyoja-dong, Nam-gu, Pohang 790-784, South Korea*

s.kettemann@jacobs-university.de

\begin{abstract}
We study the (Anderson) metal-insulator transition (MIT) in tight binding models (TBM) of disordered systems using the scaling behavior of the typical density of states (GDOS) as localization criterion. The GDOS is obtained as the geometrical mean value of the local density of states (LDOS) averaged over many different lattice sites and disorder realizations. The LDOS can efficiently be obtained within the kernel polynomial method (KPM). To check the validity and accuracy of the method, we apply it here to the standard Anderson model of disordered systems, for which the results (for instance for the critical disorder strength of the Anderson transition) are well known from other methods.
\end{abstract}

Keywords: metal-insulator transition; typical density of states; local density of states; finite-size scaling; kernel polynomial method.

PACS numbers: 71.23.An, 71.30.+h, 72.20.Ee, 64.60.an, 71.23.-k

\section{Model}

We study the well known Anderson model of a disordered system ${ }^{1}$,

$$
\hat{H}=\sum_{i} \epsilon_{i}|i\rangle\left\langle i\left|+t \sum_{\substack{i, j \\(\mathrm{NN})}}\right| j\right\rangle\langle i| .
$$

*present address 
Here, $i$ denotes the lattice sites of a 3-dimensional simple-cubic lattice, for which we consider a finite system consisting of $N=L^{3}$ sites with periodic boundary conditions; as usual, the site-diagonal matrix elements $\epsilon_{i}$ are considered to be (uncorrelated) random variables, drawn from a finite interval of width $W$ (box distribution), and the fixed (non-random) hopping matrix element $t$ is assumed to be non-vanishing for nearest neighbor sites only.

Though the localization properties of the Anderson model (critical disorder $W_{\mathrm{c}}$, scaling behavior, etc.) are well known already from the literature ${ }^{2}$, we consider it here once more to validate and test our numerical methods, which shall be applied to more realistic models in the future.

\section{Local density of states and its arithmetic and geometric average}

A physical quantity which is suitable to distinguish between localized and extended eigenstates is the local density of states (LDOS) at lattice site $i$,

$$
\rho_{i}(E)=\sum_{k=1}^{N}|\langle i \mid k\rangle|^{2} \delta\left(E-E_{k}\right),
$$

where $|k\rangle$ denotes the eigenstate with eigenenergy $E_{k}$. It is intuitively clear that $\rho_{i}(E)$ is strongly fluctuating from site to site, if the states with eigenenergy near $E$ are localized, as then only a few sites have a non-vanishing $\rho_{i}(E)>0$. Therefore, to distinguish between energy regions of localized and delocalized states, one can calculate $\rho_{i}(E)$ for many different realizations of the disordered system (or, which should be equivalent, for different sites of a given realization) and calculate the arithmetic average of the local density of states (ADOS)

$$
\rho_{\text {tot }}(E)=\frac{1}{S} \sum_{i=1}^{S} \rho_{i}(E),
$$

which corresponds to the (averaged) total density of states, and the geometric average of the local density of states (GDOS)

$$
\rho_{\text {typ }}(E)=\exp \frac{1}{S} \sum_{i=1}^{S} \log \rho_{i}(E) .
$$

which corresponds analogously to the so-called typical density of states $^{3} . S$ is the number of sites $i$ considered (which may be further increased by studying different realizations of the random system).

In the thermodynamic limit $N \rightarrow \infty$ the GDOS approaches 0 for energies $E$ in the region of (exponentially) localized states. Therefore, an investigation of the scaling behavior of the GDOS with increasing system size $N$ (or $L$ ) can be used to distinguish between energy regions of localized and delocalized states and to determine the mobility edges ${ }^{4}$ (ME) and the critical disorder strength $W_{\mathrm{c}}$ at which all states become localized (Anderson transition). 

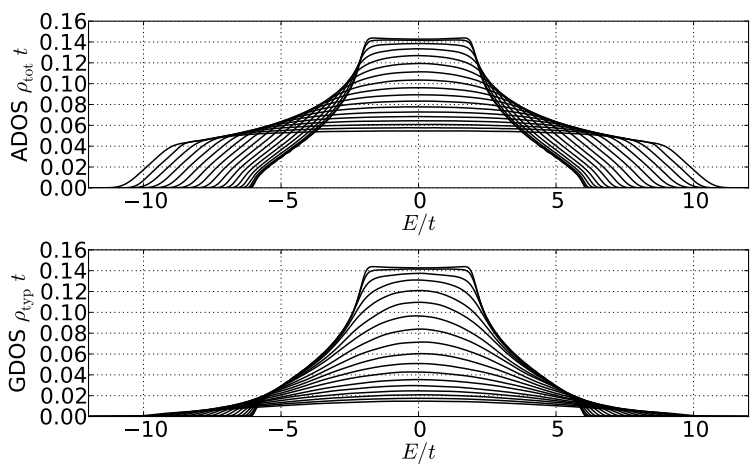

Fig. 1. The total (ADOS) and the typical (GDOS) density of states for different disorder parameters $W / t \in\{0,1,2, \ldots, 17\}$ (from top to bottom). System size is $N=40^{3}=64000$ and truncation limit is $M=140$ (see section 3 ).

The GDOS equals the ADOS for zero disorder. Otherwise it is, in general, smaller than the ADOS. Furthermore, it decreases with increasing disorder strength $W$ (see fig. 1), system size $N$, or truncation limit $M$ (see section 3 ).

\section{The kernel polynomial method}

Calculating the LDOS of tight-binding models (TBM) can most conveniently be done using the kernel polynomial $\operatorname{method}^{5}(\mathrm{KPM})$, which is based on a polynomial series expansion using Chebychev polynomials,

$$
f(x)=\frac{1}{\pi \sqrt{1-x^{2}}}\left(\mu_{0}+2 \sum_{n=1}^{\infty} \mu_{n} T_{n}(x)\right) .
$$

Truncating this series after a finite number of moments $M$ (truncation limit), the target function $f(x)$ may be approximated. The Chebychev polynomials are given by

$$
T_{n}(x)=\cos (n \arccos (x)) .
$$

In the case of the LDOS at site $i\left(f(x) \equiv \rho_{i}(E)\right)$, the coefficients $\mu_{n}$ (Chebychev moments) read

$$
\mu_{n}^{(i)}=\int_{-1}^{1} f(x) T_{n}(x) \mathrm{d} x=\left\langle i\left|T_{n}(H)\right| i\right\rangle .
$$

Recurrence relations for the $\mu_{n}$ exist $^{5}$, which can easily be implemented numerically. The core of the iteration loop consists in a sparse matrix multiplication, hence memory consumption is quite low. Another advantage of the KPM is that, given an $N \times N$ sparse matrix representation of the hamiltonian $\hat{H}(1)$, the algorithm is an $O(N)$-method, i.e. it scales linearly with $N$, which means it shows good performance even for larger system sizes. By calculating the LDOS one avoids a complete diagonalization of $H$, which would scale as $O\left(N^{3}\right)$. 


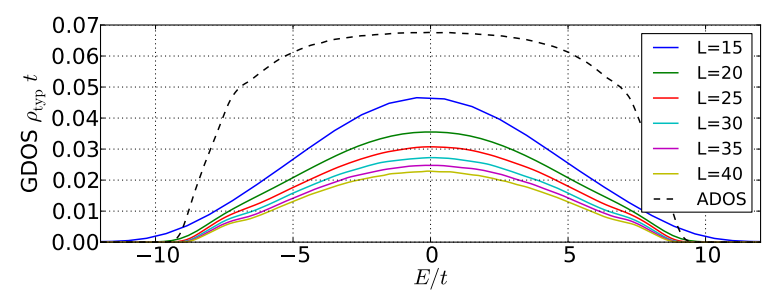

Fig. 2. Typical density of states (GDOS) for different system sizes $N$ (disorder: $W / t=13.2$ ).

\section{Distinction between localized and extended states}

As stated above, the scaling behavior of the GDOS as a function of the system size $N$ (or system edge length $L$ ) shall be used as the localization criterion to decide in which energy region the states are localized or extended (i.e., to determine the MEs). For any finite system size, the GDOS also stays finite (even for localized states) and there is no sharp but only a gradual transition between extended and localized states. Fig. 2 shows the GDOS obtained for a fixed disorder strength $(W / t=13.2)$ and different system sizes $N=L^{3}$; for comparison, the ADOS is also shown. The GDOS decreases with increasing $L$ and should approach 0 in the energy region of the localized eigenstates but a finite value in the region of delocalized states.

Therefore, the most simple ansatz for the scaling behavior of the GDOS is

$$
\rho_{\text {typ }}(E, L)=\frac{a}{L^{p}}+b .
$$

Here, the parameters $a$ and $b$ and the exponent $p$ must depend on the energy $E$. In the localized regime one has to expect $b=0$ and a large exponent $p$. Fig. 3 shows the $L$-dependence of the GDOS for different energies $E$ and again the disorder value $W / t=13.2$.

If the ratio $M / L^{d}$ ( $d=3$ for a $3 \mathrm{D}$ system) is kept constant for every investigated system size, it can be shown that directly at the transition the GDOS behaves like $1 / L$, i.e. one can expect $p=1$ exactly at the MEs. On the insulating side of the transition the scaling exponent increases $(p>1)$, and the GDOS value extrapolated for infinite system size is $b=0$. On the metallic side, the exponent apparently stays roughly at $p=1$, but the limit value is now finite $(b>0)$.

For convenience, we have assumed $b=0$ also on the metallic side. It is clear that the data belonging to metallic states now cannot be fitted very well anymore. However, the fit algorithm still tries to find the best possible fit, resulting in curves that have an even smaller curvature. Thus, the scaling exponent takes values $p<1$ in the metallic regime. This can be turned into an advantage, because now the MEs can easily be determined as those energies at which the exponent $p(E)$ crosses the value $p(E)=1$ (see figure 4 ).

It is our goal to eventually find a fit model that fits the scaling behavior of the GDOS in all band areas (not just at the MIT) and to define a better localization criterion, but until then, the above procedure is already capable of determining the 


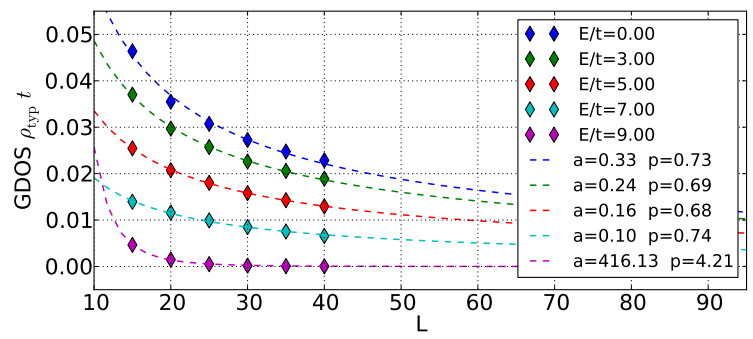

Fig. 3. Examples for the scaling behavior of the GDOS with increasing system edge length $L$ for different band energies $E$ (disorder: $W / t=13.2$ ). Resulting fit parameters from fit model (8) in the legend.

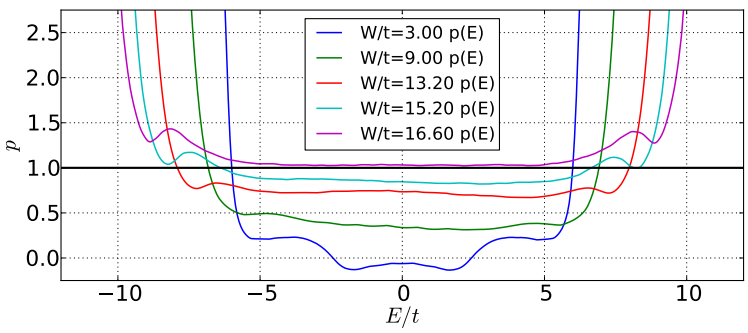

Fig. 4. Scaling exponent $p$ as a function of energy $E$ for different disorder parameters $W$, using the simple fit model with $b=0$. The thick black line indicates the cutoff $c=1$ that is used to determine the mobility edges.

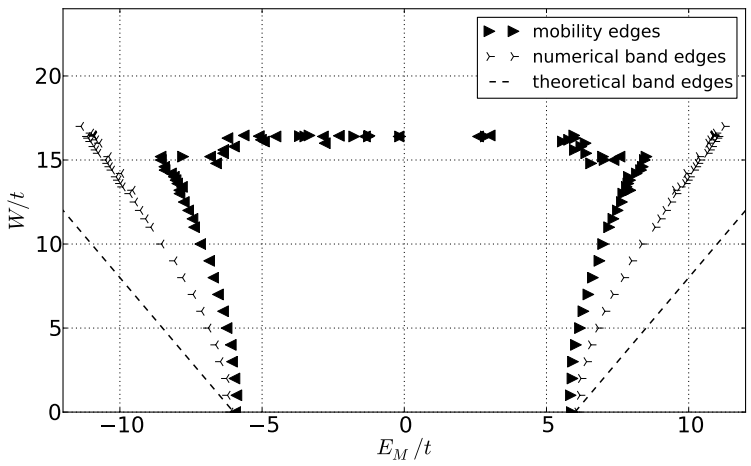

Fig. 5. Phase diagram of disorder as obtained by our method with the accuracy of our current data.

MIT for any range of disorder parameters $W$. This allows for the calculation of the phase diagram in the $W-E$ plane, which shows the MEs $E_{\mathrm{M}}$ for different disorder strengths $W$ and therefore the lines at which the MIT takes place (see figure 5).

Although the reached accuracy is still not satisfying - in particular close to the critical disorder strength $W_{\mathrm{c}} / t \approx 16.5-$, the phase diagram shown in figure 5 
already features some well-known $\operatorname{properties}^{3,5}$ :

- For zero disorder, the mobility edges coincide with the band edges.

- For increasing disorder, the energy spectrum broadens, and localized states appear in the band tails.

- Reentrance behavior: At a specific disorder parameter $W_{\mathrm{t}}$, the width between the two MEs shrinks dramatically. However, the extremal energy values reached at these turning points are slightly different from the ones in the literature ${ }^{3}$ $\left(E_{\mathrm{t}} / t \approx 8.3\right.$ instead of $\left.E_{\mathrm{t}} / t \approx 7.6\right)$.

- The critical disorder is reached at about $W_{\mathrm{c}} / t \approx 16.5$.

\section{Conclusion}

We have shown that even with the very crude and oversimplified ansatz (8) for the scaling law of the GDOS, good results for the MEs and the Anderson MIT and a reasonable estimate for the phase diagram can be obtained. However, to avoid fluctuations in the scaling exponent $p(E)$ (and thus in the position of the MEs), either the accuracy has to be further increased (especially near the critical disorder $W_{\mathrm{c}}$ ), or a more sophisticated fit model has to be developed. Under the given circumstances, we are confident to be able to develop a method which does not rely on prior knowledge of external calibration parameters (e.g. the position of the critical disorder $W_{\mathrm{c}}$ ) in the near future.

After successful implementation of the algorithms and application to the Anderson model (1), we want to apply the procedure to more interesting and more realistic systems, e.g. binary alloys and multi-band TBMs, describing, for instance, semiconductors with magnetic impurities.

\section{References}

1. P. W. Anderson, Phys. Rev. 109, 1492 (1958).

2. B. Kramer, A. MacKinnon, Rep. Prog. Phys. 56, 1469 (1993).

3. G. Schubert and H. Fehske, Quantum Percolation in Disordered Structures, in Quantum and Semiclassical Percolation and Breakdown in Disordered Solids, Lecture Notes in Physics, Vol. 762/2009 (Springer, Berlin/Heidelberg, 2009), p. 1-28.

4. N. Mott, Physics Today 31, 42 (1978).

5. A. Weiße et al., Rev. Mod. Phys. 78, 275-306 (2006). 